\title{
Tobacco smoking relapse in post-partum period
}

Marie Blanquet, Anne Debost-Legrand, Marion Arnoult and Françoise Vendittelli 


\section{Tobacco during pregnancy}

- In France, prevalence of tobacco consumption (2016)

- Before pregnancy $30.0 \%$

- During the $3^{\text {rd }}$ trimester $16.6 \%$

- Smoking cessation during pregnancy

- $45 \%$ spontaneous cessation

- $16 \%$ during the $1^{\text {st }}$ trimester

- Screening of tobacco consumption by health professionnals not systematic ( $80 \%$ of pregnant women)

- Brief intervention for smoking cessation performed for $46.3 \%$ of smoking pregnant women 


\section{Smoking cessation in post-partum}

- High rate of smoking cessation failure in post-partum

- $18.5-58.0 \%$ at 3 months

- $28.9-70.5 \%$ at 6 months

- Main risk factors of smoking cessation failure identified

- Tobacco status of the partner

- Stress events

- Depressive symptoms in post-partum

- Social deprivation

- Low level of education

- Protective factor: breastfeeding 


\section{Objectives}

- Main objective

To assess prevalence of smoking lapse and relapse in post-partum period

- Secondary objectives

To identify risk factors of smoking lapse and relapse 


\section{Methods}

-Women who stopped smoking 3 months before or during pregnancy

- Three time period cross-sectionnal study: M0,M3,M6 from september 2016 to january 2017

- Self questionnaire:

- EPICES score (Social deprivation)

- Heavy Smoking Index (HSI)

- Tobacco Craving Questionnaire in treatment-seeking smokers (FTCQ12)

- Edinburgh Postnatal Depression Scale (EPDS)

- Main evaluation criteria:

- Lapse: less than 1 cigarette per day on 7 days

- Relapse: at least 1 cigarette par day on 7 days 


\section{Results (1)}

Women characteristics at M0

\begin{tabular}{cc}
\hline & $\% \mathrm{~m}[\mathrm{sd}](\mathrm{N})$ \\
\hline Age & $30.8[4.8](100)$ \\
\hline Marital status (in couple) & $90.9(99)$ \\
\hline Level of education (high) & $44.1(93)$ \\
\hline Working status (active) & $76.9(91)$ \\
Social deprivation (EPICES $\geq 30.17)$ & $25.5(94)$ \\
EPDS ( $\geq 12)$ & $35.7(98)$ \\
\hline Breastfeeding & $61.3(62)$ \\
\hline
\end{tabular}

Tobacco consumption characteristics

\begin{tabular}{cc}
\hline & $\% \mathrm{~m}[\mathrm{sd}] \mathrm{(N)}$ \\
\hline Tobacco dependence before cessation (HSI) & $(100)$ \\
Very low & 81.0 \\
Low & 13.0 \\
Moderate & 5.0 \\
High & 1.0 \\
\hline Craving (FTCQ12, global score) & $2.1[0.9](99)$ \\
Smoking cessation & $(100)$ \\
Before pregnancy & 11.0 \\
1st trimester & 73.0 \\
\hline Smoking cessation supervised by tobaccologist & $6.0(6)$ \\
Specific treatment & $10.0(10)$ \\
\hline Partner Smoking status (smoker) & $60.4(96)$ \\
\hline
\end{tabular}




\section{Results (2)}

Prevalence of smoking relapse during post-partum

\begin{tabular}{ccc}
\hline & M3 & M6 \\
\hline Total Smoker & $38.7(63)$ & $37.8(37)$ \\
Lapse & $29.2(24)$ & $38.5(13)$ \\
Relapse & $70.8(24)$ & $31.5(8)$ \\
Craving & $3.2[0.9](22)$ & $3.4[1.5](14)$ \\
(FTCQ12) & & \\
\hline
\end{tabular}

Risk factors of smoking relapse during post-partum

\begin{tabular}{lcccc}
\hline & Hazard Ratio & 95\% IC & p \\
\hline No breastfeeding & 3.6 & 1.4 & 8.6 & 0.0042 \\
$\begin{array}{l}\text { Craving at M3 } \\
\text { (FTCQ12) }\end{array}$ & 2.1 & 1.4 & 3.1 & 0.0002 \\
\hline
\end{tabular}

Trend of global score of FTCQ12 from M0 to M3

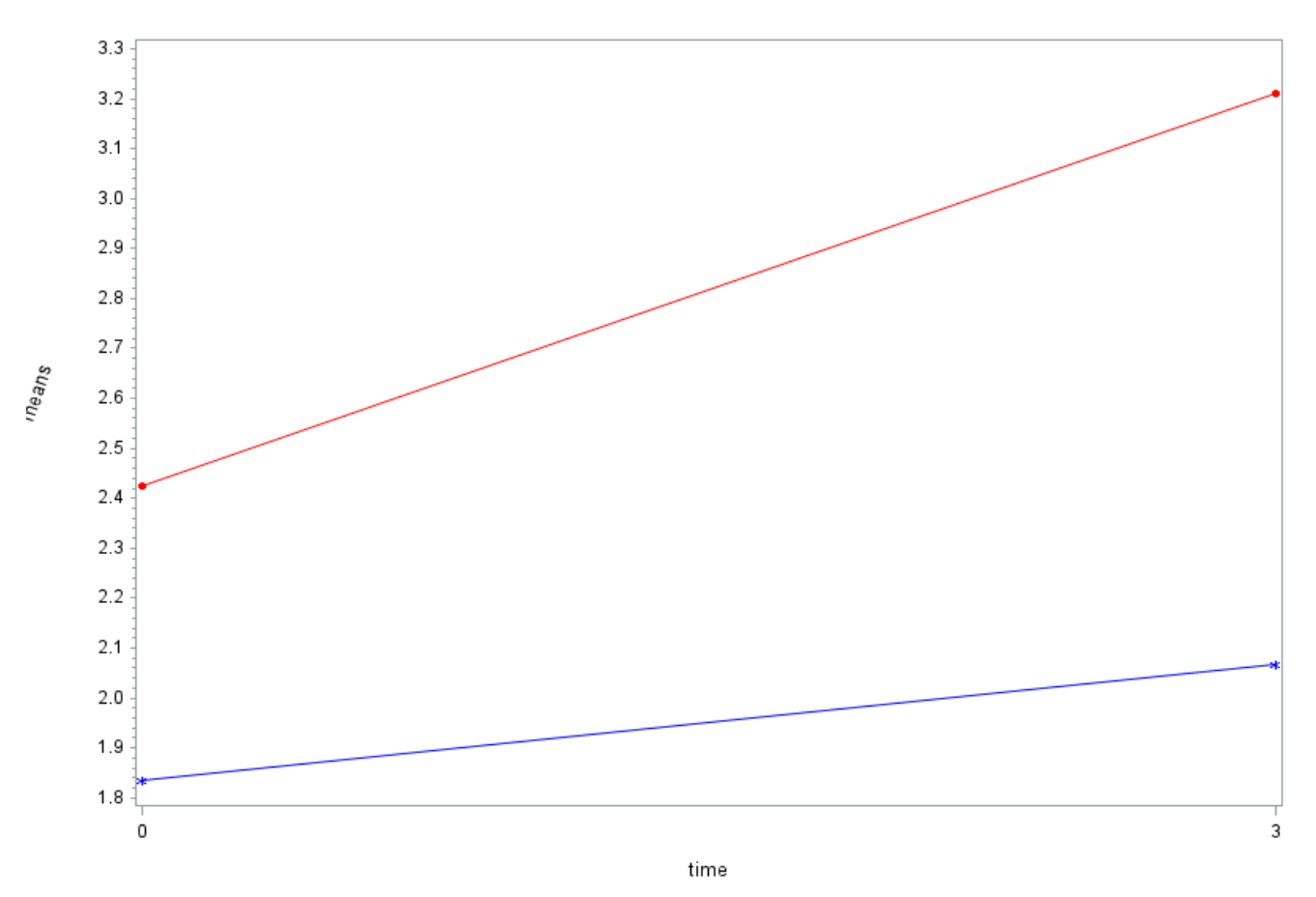




\section{Discussion and conclusion}

- High prevalence of lapse and relapse : $38.7 \%$ at $\mathrm{M} 3-37.8 \%$ at $\mathrm{M} 6$

- Low dependance among our population

- Low support of smoking cessation by health professionals

- Low use of specific treatment

- High global score of FTCQ12 in women who relapse

- Craving most frequent symptom of abstainers

- FTCQ12 use in clinical practice rapidly predicts smoking status

- Breastfeeding appears to be a protective factor

- Results validated at M3 only (many lost-of-follow up at M6)

Health professionals should aware of screening and supporting pregnant women to quit smoking 
mampRris:

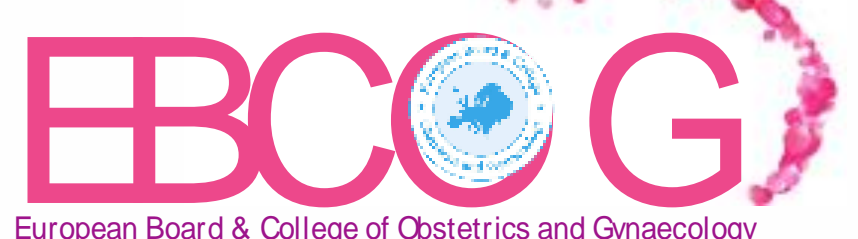

Beck

March 8-10, 2018

Thank you for your attention 